International Electronic Journal of Geometry

Volume 8 No. 1 PP. 105-115 (2015) (CIEJG

\title{
BELTRAMI-EULER FORMULAS OF GENERALIZED SEMI-RULED SURFACE IN SEMI EUCLIDEAN SPACE
}

\author{
MAHMUT AKYİĞİT, SOLEY ERSOY AND MURAT TOSUN
}

(Communicated by Levent KULA )

\begin{abstract}
In this paper, we obtain some relations related to the principal sectional curvatures of generalized semi ruled surfaces with central ruled surfaces in terms of the determinant of the first fundamental form of these surfaces. Also, we investigate the same relations at the central points of generalized semi ruled surfaces. Moreover by applying Euler theorem to tangential sections of the generalized semi ruled surfaces in n-dimensional semi Euclidean space. We find the relationship between the sectional curvatures of non-degenerate sections and the principal sections in two different type and call as $1^{\text {st }}$ and $2^{\text {nd }}$ type Semi Euclidean Beltrami-Euler formulas.
\end{abstract}

\section{INTRODUCTION}

The last three centuries have seen the theory of curvature bloom. L. Euler introduced the theory of curvature of surface in his first studies, especially in "L'application de l'analyse a la geometrie". Euler's sectional curvatures were basically curvatures of curves obtained by intersections of a normal plane of the curves. Thus, the Euler theorem (Euler-curvature formula) related to the normal and principal curvatures entered to literature. Applying Euler theorem which is a well-known theorem in the classical surface theory for the tangential sections of the generalized ruled surface was performed in [9]. Moreover, $(k+1)$-dimensional ruled surfaces in $E^{n}$ were studied in [8]-[9]. Several properties of two-dimensional ruled surfaces were also given in [11]. In the recent times, the curvatures of ruled surfaces in $E_{1}^{3}$ were studied in [13]. The sectional curvatures of the generalized ruled surfaces were evaluated in $n$-dimensional Euclidean space $E^{n}$ and the obtained relations were called Beltrami-Euler formula in [9]. Also, the sectional curvatures of timelike ruled surfaces in $E_{1}^{n}$ were studied in [5],[6],[7]. In [3], parallel timelike ruled surfaces and compare geometric invariants of two parallel ruled surfaces were defined. They obtained the geodesic curvature, the geodesic torsion of a curve and the normal curvature. The $(k+1)$-dimensional generalized semi ruled surfaces in

Date: Received: June 5, 2014 and Accepted: March 3, 2015.

2010 Mathematics Subject Classification. 14Q10, 53A35.

Key words and phrases. Sectional curvature, Ruled surface, Beltrami-Euler formula.

The study was supported by Sakarya University Scientific Research Projects Commission under the grant no: 2010-50-02-018. 
$E_{v}^{n+1}$ were given by [4]. In this work, we have studied the sectional curvatures of the generalized semi ruled surfaces in $(n+1)$-dimensional semi Euclidean space $E_{v}^{n+1}$. Furthermore, we have obtained the semi-Euclidean Beltrami-Euler formula in $(n+1)$-dimensional semi-Euclidean space $E_{v}^{n+1}$.

Semi Euclidean space $E_{v}^{n+1}$ is an Euclidean space provided with the metric tensor

$$
d s^{2}=\sum_{i=1}^{n-\nu} d x_{i}^{2}-\sum_{i=n-\nu+1}^{n+1} d x_{i}^{2}
$$

where $\left\{x_{1}, \ldots, x_{n}, x_{n+1}\right\}$ is the rectangular coordinate systems of $E_{v}^{n+1}$. Especially, if $v=0$, then $E_{0}^{n+1}$ is called Euclidean space. If $v=1$ and $n \geq 2, E_{1}^{n+1}$ is called as Minkowski $(n+1)$-space, [10]. Since $d s^{2}$ is an indefinite metric, recall that a vector $x \in E_{v}^{n+1}$ can have one of the three causal characters; it can be spacelike if $d x^{2}>0$ or $x=0$, timelike if $d x^{2}<0$ and null(lightlike) if $d x^{2}=0$ and $x \neq 0$.

\section{Generalized Semi-Ruled Surface in $E_{v}^{n+1}$}

Let $\alpha$ be a differentiable curve

$$
\begin{aligned}
\alpha: I & \rightarrow E_{v}^{n+1} \\
t & \rightarrow \alpha(t)=\left(\alpha_{1}(t), \alpha_{2}(t), \ldots, \alpha_{n}(t), \alpha_{n+1}(t)\right)
\end{aligned}
$$

in the $(n+1)$-dimensional semi-Euclidean space $E_{v}^{n+1}$ where $\{0\} \subset I \subset R$.

An orthonormal vector system $\left\{e_{1}(t), e_{2}(t), \ldots, e_{k}(t)\right\}$ defined at each point $\alpha(t)$ of the curve $\alpha$ spans a subspace of the tangent space $T_{E_{v}^{n+1}}(\alpha(t))$ at $\alpha(t) \in E_{v}^{n+1}$. If this subspace is denoted by $E_{k, \mu}(t)$, then

$$
E_{k, \mu}(t)=S p\left\{e_{1}(t), e_{2}(t), \ldots, e_{k}(t)\right\} \subset E_{v}^{n+1}, \quad 0 \leq \mu \leq v .
$$

This subspace is called as semi-subspace and

$$
\left\langle e_{i}(t), e_{j}(t)\right\rangle=\varepsilon_{i} \delta_{i j} \quad, \quad \varepsilon_{i}=\left\{\begin{aligned}
1, & 1 \leq i \leq k-\mu \\
-1, & k-\mu+1 \leq i \leq k .
\end{aligned}\right.
$$

For $\mu \geq 1$, there are $\mu$ timelike vectors in the semi-subspace $E_{k, \mu}(t)$. If there is no timelike vector field in $E_{k, \mu}(t)$, then $E_{k, 0}(t)=E_{k}(t)$ is an Euclidean subspace. If there is one timelike vector in $E_{k, \mu}(t)$, then $E_{k, 1}(t)$ is a timelike subspace.

While the semi-subspace $E_{k, \mu}(t)$ is moving through the curve $\alpha, E_{k, \mu}(t)$ generates a $(k+1)$-dimensional surface. This surface is called $(k+1)$-dimensional generalized semi-ruled surface in $(n+1)$-dimensional semi-Euclidean space $E_{v}^{n+1}$ and is denoted by $M$. In addition that, $M$ can be expressed by the following parametric equation

$$
\varphi\left(t, u_{1}, \ldots u_{k}\right)=\alpha(t)+\sum_{i=1}^{k} u_{i} e_{i}(t) \quad, \quad\left(t, u_{1}, \ldots u_{k}\right) \in I \times \Re^{k} .
$$

Here, the subspace $E_{k, \mu}(t)$ and the curve $\alpha$ are called the generating space and base curve, respectively, [4].

Throughout this paper, we assume that $E_{k, \mu}(t), \mu \geq 1$, is a semi-subspace and $\left\{\dot{\alpha}(t)+\sum_{i=1}^{k} u_{i} \dot{e}_{i}(t), e_{1}(t), \ldots, e_{k}(t)\right\}$ is linearly independent and the base curve $\alpha$ is a non-null curve, [4]. 
Let $\dot{e}_{i}(t)$ be the velocity of the vector fields of $e_{i}(t), 1 \leq i \leq k$, along the base curve $\alpha$, thus the subspace

$$
A(t)=S p\left\{e_{1}, \ldots, e_{k}, \dot{e_{1}}, \ldots, \dot{e_{k}}\right\}
$$

is called as asymptotic bundle of $M$ with respect to $E_{k, \mu}(t)$.

If $\operatorname{dim} A(t)=k+m, 0 \leq m \leq k$, then one can find an orthonormal base for $A(t)$ containing $E_{k, \mu}(t)$ such as

$$
\left\{e_{1}(t), \ldots, e_{k}(t), a_{k+1}(t), \ldots, a_{k+m}(t)\right\} .
$$

It is clear that $A(t)$ is a semi-subspace. Also, for the orthonormal base $\left\{e_{1}(t)\right.$, $\left.e_{2}(t), \ldots, e_{k}(t)\right\}$ there are the following equations, [4],

$$
\begin{array}{ll}
\dot{e_{i}}=\sum_{j=1}^{k} \alpha_{i j} e_{j}+\varepsilon_{k+i} \kappa_{i} a_{k+i} & , \quad 1 \leq i \leq m \\
\dot{e_{h}}=\sum_{j=1}^{k} \alpha_{h j} e_{j} & ,
\end{array}
$$

where

$$
\varepsilon_{i j} \alpha_{i j}=-\alpha_{j i}, \varepsilon_{j}=\left\langle e_{j}, e_{j}\right\rangle, \varepsilon_{i j}=\varepsilon_{i} \varepsilon_{j}
$$

and for $r \leq \mu$,

$$
\begin{aligned}
& \kappa_{1}>\kappa_{2}>\ldots>\kappa_{m-r}>0 \\
& \kappa_{m-r+1}<\kappa_{m-r+2}<\ldots<\kappa_{m}<0 .
\end{aligned}
$$

The subspace

$$
S p\left\{e_{1}(t), \ldots, e_{k}(t), \dot{e_{1}}(t), \ldots, \dot{e_{k}}(t), \dot{\alpha}(t)\right\}
$$

is called tangential bundle of $M$ with respect to $E_{k, \mu}(t)$ and is denoted by $T(t)$. It is obvious that $k+m \leq \operatorname{dim} T(t) \leq k+m+1,0 \leq m \leq k$.

If $\operatorname{dim} T(t)=k+m$, then $A(t)=T(t)$, that is, the tangential bundle $T(t)$ is a semi-subspace. If $\operatorname{dim} T(t)=k+m+1$, due to $\dot{\alpha} \notin A(t)$, then an orthonormal base can be found for $T(t)$ as

$$
\left\{e_{1}, \ldots, e_{k}, a_{k+1}, \ldots, a_{k+m}, a_{k+m+1}\right\} .
$$

The tangential bundle $T(t)$ has at least number of $\mu$ timelike vectors. Therefore, $T(t)$ is a semi-subspace, [4].

If $\operatorname{dim} T(t)=k+m+1$, then $(k+1)$-dimensional semi-ruled surface $M$ has $(k-m)$ - dimensional subspace called central space of $M$ and this subspace is denoted by $Z_{k-m, r}(t) \subset E_{k, \mu}(t)$. It is clear that $Z_{k-m, r}(t) \subset E_{k, \mu}(t)$ is a semisubspace. While semi-subspace $Z_{k-m, r}(t)$ is moving through the base curve $\alpha$ of $M$, it generates a $(k-m+1)$-dimensional ruled surface contained by $M$. This surface is called as $(k-m+1)$-dimensional central ruled surface and denoted by $\Omega$. Since $Z_{k-m, r}(t)$ is a semi-subspace, central ruled surface $\Omega$ is also a semi-ruled surface, [4].

If we assume the base curve $\alpha$ of $(k+1)$ - dimensional semi-ruled surface $M$ is also base curve of $\Omega \subset M$, then 


$$
\dot{\alpha}(t)=\sum_{\nu=1}^{k} \zeta_{\nu} e_{\nu}+\eta_{m+1} a_{k+m+1} \quad, \quad \eta_{m+1} \neq 0 .
$$

The tangential space of $M$ is perpendicular to the asymptotic bundle $A(t)$ at the central points. Taking the equation (2.5) at the central point of central ruled surface $\Omega \subset M$, we see that [4].

$$
u_{\sigma}=0 \quad, \quad 1 \leq \sigma \leq m .
$$

Let $\beta$ be an orthogonal trajectory of central semi-ruled surface $\Omega$ and the subspace $F_{m, h}(t),(h \leq r)$ be as totaly perpendicular to the generating space $Z_{k-m, r}(t)$. While $F_{m, h}(t)$ subspace is moving through the curve $\beta$, it generates a $(m+1)-$ dimensional ruled surface. This ruled surface is called principal ruled surface of $M$ and is denoted by $\Lambda$. Since $F_{m, h}(t)$ is semi-subspace, principal ruled surface $\Lambda$ is also semi-ruled space. For the velocity vector of base curve of semi-ruled space given by equation (2.5), the magnitude

$$
P_{i}=\frac{\eta_{m+1}}{\kappa_{i}} \quad, \quad 1 \leq i \leq m
$$

is called the $i^{\text {th }}$ principal distribution parameter of $M$.

The canonical base of tangential bundle of $M$ in $E_{v}{ }^{n+1}$ is

$$
\left\{\sum_{i=1}^{k}\left(\zeta_{i}+\sum_{j=1}^{k} \alpha_{j i} u_{j}\right) e_{i}+\sum_{\sigma=1}^{m} \varepsilon_{k+\sigma} u_{\sigma} \kappa_{\sigma} a_{k+\sigma}+\eta_{m+1} a_{k+m+1}, e_{1}, e_{2}, \ldots, e_{k}\right\} .
$$

Then, we can evaluate the first fundamental form of $M$ and the metric coefficients with respect to this canonical base. For a conventional notation, we choose $u_{0}=t$ and calculate the metric coefficients of $M$ as follows

$$
\begin{array}{ll}
g_{00}=\left\langle\varphi_{t}, \varphi_{t}\right\rangle=\sum_{i=1}^{k} \varepsilon_{i}\left(\zeta_{i}+\sum_{j=1}^{k} u_{j} \alpha_{j i}\right)^{2}+\sum_{\sigma=1}^{m} \varepsilon_{k+\sigma}\left(u_{\sigma} \kappa_{\sigma}\right)^{2}+\varepsilon_{k+m+1}\left(\eta_{m+1}\right)^{2} \\
g_{i 0}=\left\langle\varphi_{u_{i}}, \varphi_{t}\right\rangle=\varepsilon_{i}\left(\zeta_{i}+\sum_{j=1}^{k} u_{j} \alpha_{j i}\right) & , \quad 1 \leq i \leq k \\
g_{i j}=\left\langle\varphi_{u_{i}}, \varphi_{u_{j}}\right\rangle=\varepsilon_{i} \delta_{i j} & , \quad 1 \leq i, j \leq k .
\end{array}
$$

Therefore, the matrix of the first fundamental form of $M$ is expressed to be $\left[g_{x y}\right]$. In these regards, from the equations (2.8), it is seen that

$$
g=\operatorname{det}\left[g_{x y}\right]=\varepsilon\left(\sum_{\sigma=1}^{m} \varepsilon_{k+\sigma}\left(u_{\sigma} \kappa_{\sigma}\right)^{2}+\varepsilon_{k+m+1}\left(\eta_{m+1}\right)^{2}\right) \quad, \quad 1 \leq x, y \leq k .
$$

In addition to these, the elements of the inverse matrix of $\left[g_{x y}\right]$ are obtained to be

$$
\begin{aligned}
& g^{00}=\varepsilon g^{-1} \\
& g^{i 0}=-\varepsilon g^{-1}\left(\zeta_{i}+\sum_{j=1}^{k} u_{j} \alpha_{j i}\right), \quad 1 \leq i \leq k \\
& g^{i \lambda}=g^{-1}\left(\left(\zeta_{i}+\sum_{j=1}^{k} u_{j} \alpha_{j i}\right)\left(\zeta_{\lambda}+\sum_{j=1}^{k} u_{j} \alpha_{j \lambda}\right) \varepsilon+\delta_{i \lambda} \varepsilon_{i} g\right), 1 \leq i, \lambda \leq k
\end{aligned}
$$

where $\varepsilon=\varepsilon_{1} \cdot \varepsilon_{2} \cdot \varepsilon_{3} \ldots . \varepsilon_{k},[1]$.

Let $\left\{u_{0}, u_{1}, \ldots, u_{k}\right\}$ be a base of tangent space at the neighborhood of the coordinate systems $\left\{\partial_{0}, \partial_{1}, \ldots, \partial_{k}\right\},\left(\frac{\partial}{\partial u_{i}}=\partial_{i}, 0 \leq i \leq k\right)$ of $M$, then the Riemannian curvature tensor of $M$ is 


$$
R_{\partial_{i} \partial_{j}}\left(\partial_{l}\right)=\sum_{r=0}^{k} R_{l i j}^{r} \partial_{r}
$$

where the coefficient of the Riemannian curvature tensor is

$$
R_{l i j}^{r}=\frac{\partial}{\partial u_{i}} \Gamma_{j l}^{r}-\frac{\partial}{\partial u_{j}} \Gamma_{i l}^{r}-\sum_{s=0}^{k} \Gamma_{i l}^{s} \Gamma_{j s}^{r}+\sum_{s=0}^{k} \Gamma_{j l}^{s} \Gamma_{i s}^{r} .
$$

Therefore, the Riemannian-Christoffel curvature tensor of $M$ becomes

$$
R_{h l i j}=\sum_{r=0}^{k} g_{r h}\left(\frac{\partial}{\partial u_{i}} \Gamma_{j l}^{r}-\frac{\partial}{\partial u_{j}} \Gamma_{i l}^{r}-\sum_{s=0}^{k} \Gamma_{i l}^{s} \Gamma_{j s}^{r}+\sum_{s=0}^{k} \Gamma_{j l}^{s} \Gamma_{i s}^{r}\right) .
$$

Moreover, there exist the following relations, [4].

$$
\begin{aligned}
& R_{h l x y}=R_{x y h l} \\
& R_{x y h l}=-R_{y x h l} .
\end{aligned}
$$

Considering Christoffel symbols and the equation (2.11), $R_{i j 00}, R_{i j \nu \mu}, R_{\nu 0 \mu 0}$ are found to be

$$
\begin{array}{ll}
R_{i j 00}=0 & , \quad 0 \leq i, j \leq k \\
R_{i j x y}=0 & , \quad 0 \leq i, j \leq k, 1 \leq x, y \leq k, \\
R_{x 0 y 0}=\varepsilon\left(\frac{1}{4 g} \frac{\partial g}{\partial u_{x}} \frac{\partial g}{\partial u_{y}}-\frac{1}{2} \frac{\partial^{2} g}{\partial u_{x} \partial u_{y}}\right) \quad, \quad 1 \leq x, y \leq k,
\end{array}
$$

$[1]$

\section{Beltrami-Euler Formula for Generalized Semi-Ruled Surfaces in Semi-Euclidean Space, $E_{v}^{n+1}$}

Two-dimensional subspace $\Pi$ of $(k+1)$-dimensional semi-ruled surface at the point $\xi \in M$ is called tangent section of $M$. If $v$ and $w$ form a basis of the tangent section $\Pi$, then $Q(v, w)=\langle v, v\rangle\langle w, w\rangle-\langle v, w\rangle^{2}$ is a nonzero quantity if and only if $\Pi$ is non-degenerate. The quantity $|Q(\vec{v}, \vec{w})|$ represents the square of the semiEuclidean area of the parallelogram determined by $v$ and $w$. Using the square of the semi-Euclidean area of the parallelogram determined by the basis vectors $\{v, w\}$, one has the following classification for the tangent sections of the semi-ruled surfaces

$$
\begin{array}{ll}
Q(v, w)=\langle v, v\rangle\langle w, w\rangle-\langle v, w\rangle^{2}<0 \quad, \quad & \text { (timelike plane), } \\
Q(v, w)=\langle v, v\rangle\langle w, w\rangle-\langle v, w\rangle^{2}=0 \quad, \quad & \text { (degenerate plane), } \\
Q(v, w)=\langle v, v\rangle\langle w, w\rangle-\langle v, w\rangle^{2}>0 \quad, \quad & \text { (spacelike plane). }
\end{array}
$$

For the non-degenerate tangent section $\Pi$ given by the basis $\{v, w\}$ of $M$ at the point $\xi$, the sectional curvature of $M$ at the point $\xi$ is defined by

or

$$
K_{\xi}(v, w)=\frac{\left\langle R_{v w} v, w\right\rangle}{\langle v, v\rangle\langle w, w\rangle-\langle v, w\rangle^{2}}
$$

$$
K(v, w)=\frac{\sum R_{i j k m} w_{i} v_{j} w_{k} v_{m}}{\sum g_{i j} v_{i} v_{j} g_{k m} w_{k} w_{m}-\left[\sum g_{i j} v_{i} w_{j}\right]^{2}}
$$


where $v=\sum \beta_{i} \frac{\partial}{\partial x_{i}}$ and $w=\sum \gamma_{j} \frac{\partial}{\partial x_{j}}$. Here $R$ is a $(1,3)$-tensor field on $M$ and the coordinates of the basis vectors $v$ and $w$ are $\left(\beta_{0}, \beta_{1}, \ldots, \beta_{k}\right)$ and $\left(\gamma_{0}, \gamma_{1}, \ldots, \gamma_{k}\right)$, respectively, [2].

Let the base curve $\alpha$ of ruled surface $M$ with non-degenerate generating space be also a base curve of central ruled surface $\Omega$ of $M$ in $E_{v}^{n+1}$. In this case, a normal tangent vector $n$ of $M$ at the point $\forall \xi\left(t, u_{\nu}\right)$ which is orthogonal to $E_{k, \mu}(t)$ is defined to be

$$
n=\sum_{\sigma=1}^{m} u_{\sigma} \kappa_{\sigma}(t) a_{k+\sigma}(t)+\eta_{m+1} a_{k+m+1}(t) \quad, \quad\left(\eta_{m+1} \neq 0\right) .
$$

The normal tangent vector field is always non-null vector.

Considering the equations (3.1) and (3.3), the following theorem related to principal sectional curvature at the point $\xi \in M$ can be given.

Theorem 3.1. Let $M$ be a generalized semi-ruled surface with central semi-ruled surface in $(n+1)$-dimensional semi-Euclidean space $E_{v}^{n+1}$. For non-degenerate (spacelike or timelike) normal tangential vector $n$, the principal sectional curvature $\left(e_{i}, n\right), 1 \leq i \leq k$ is given by

$$
K_{\xi}\left(e_{x}, n\right)=\varepsilon_{x}\left(-\frac{1}{2 g} \frac{\partial^{2} g}{\partial u_{x}^{2}}+\frac{1}{4 g^{2}}\left(\frac{\partial g}{\partial u_{x}}\right)^{2}\right), \quad 1 \leq x \leq k
$$

at the point $\xi \in M$.

Proof. Let $\left(\beta_{0}, \beta_{1}, \ldots, \beta_{k}\right)$ and $\left(\gamma_{0}, \gamma_{1}, \ldots, \gamma_{k}\right)$ be, respectively, the coordinates of $e_{i}, 1 \leq i \leq k$ and $n$, which form the basis of the principal section $\left(e_{i}, n\right)$, with respect to canonical basis given by the equation (2.7). Considering equations (3.1), we find that the $i^{\text {th }}$ principal sectional curvature of $\left(e_{i}, n\right),(1 \leq i \leq k)$ as

$$
K_{\xi}\left(e_{i}, n\right)=\frac{\beta_{i} \beta_{i} \gamma_{0} \gamma_{0} R_{i 0 i 0}}{\left\langle e_{i}, e_{i}\right\rangle\langle n, n\rangle-\left\langle e_{i}, n\right\rangle^{2}}
$$

If we substitute the equations (2.13) and (3.3) into the last equation, we obtain the $i^{\text {th }}$ principal sectional curvature

$$
K_{\xi}\left(e_{i}, n\right)=\frac{\varepsilon\left(-\frac{1}{2} \frac{\partial^{2} g}{\partial u_{i}^{2}}+\frac{1}{4 g}\left(\frac{\partial g}{\partial u_{i}}\right)^{2}\right)}{\left\|e_{i}\right\|^{2}\left\|\sum_{\sigma=1}^{m} u_{\sigma} \varepsilon_{k+\sigma} \kappa_{\sigma} a_{k+\sigma}+\eta_{m+1} a_{k+m+1}\right\|^{2}-\left\langle e_{i}, \sum_{\sigma=1}^{m} u_{\sigma} \varepsilon_{k+\sigma} \kappa_{\sigma} a_{k+\sigma}+\eta_{m+1} a_{k+m+1}\right\rangle^{2}}
$$

where $1 \leq i \leq k, 1 \leq \sigma \leq m$.

If we consider $\varepsilon_{i}=\left\langle e_{i}, e_{i}\right\rangle, \varepsilon=\varepsilon_{1} \cdot \varepsilon_{2} \ldots . \varepsilon_{k}$, this last equation gives us the $i^{\text {th }}$ principal sectional curvature as

$$
K_{\xi}\left(e_{i}, n\right)=\frac{\varepsilon\left(-\frac{1}{2} \frac{\partial^{2} g}{\partial u_{i}^{2}}+\frac{1}{4 g}\left(\frac{\partial g}{\partial u_{i}}\right)^{2}\right)}{\varepsilon_{i} \cdot \varepsilon g} .
$$

By substituting the equation (2.8) and the equality

$$
g=\varepsilon\left(\sum_{\sigma=1}^{m} \varepsilon_{k+\sigma}\left(u_{\sigma} \kappa_{\sigma}\right)^{2}+\varepsilon_{k+m+1}\left(\eta_{m+1}\right)^{2}\right)
$$

into the last equation and rearranging it, the equation (3.4) is found and the proof is completed. 
Theorem 3.2. Let $M$ be a generalized semi-ruled surface with central semi-ruled surface and $n$ be non-null tangential vector in $(n+1)$-dimensional semi-Euclidean space $E_{v}^{n+1}$. The $\sigma^{\text {th }}$ principal sectional curvature and the $(m+\rho)^{\text {th }}$ principal sectional curvature of $M$ at the point $\xi \in M$ are

$$
K_{\xi}\left(e_{\sigma}, n\right)=\varepsilon_{\sigma} \kappa_{\sigma}^{2}\left(\frac{\left(u_{\sigma} \kappa_{\sigma}\right)^{2}-\varepsilon_{k+\sigma}\left(\sum_{\sigma=1}^{m} \varepsilon_{k+\sigma}\left(u_{\sigma} \kappa_{\sigma}\right)^{2}+\varepsilon_{k+m+1}\left(\eta_{m+1}\right)^{2}\right)}{\left(\sum_{\sigma=1}^{m} \varepsilon_{k+\sigma}\left(u_{\sigma} \kappa_{\sigma}\right)^{2}+\varepsilon_{k+m+1}\left(\eta_{m+1}\right)^{2}\right)^{2}}\right), 1 \leq \sigma \leq m,
$$

and

$$
K_{\xi}\left(e_{m+\rho}, n\right)=0 \quad, \quad 1 \leq \rho \leq k-m,
$$

respectively, where $\varepsilon_{\sigma}=\left\langle e_{\sigma}, e_{\sigma}\right\rangle= \pm 1, \varepsilon_{k+m+1}=\left\langle a_{k+m+1}, a_{k+m+1}\right\rangle= \pm 1, \varepsilon_{k+\sigma}=$ $\left\langle a_{k+\sigma}, a_{k+\sigma}\right\rangle= \pm 1$.

Proof. Considering the equation (2.8), we see that

$$
\begin{gathered}
\frac{\partial g}{\partial u_{\sigma}}=2 \varepsilon \varepsilon_{k+\sigma} u_{\sigma} \kappa_{\sigma}^{2} \quad, \quad \frac{\partial^{2} g}{\partial u_{\sigma}^{2}}=2 \varepsilon \varepsilon_{k+\sigma} \kappa_{\sigma}^{2} \\
\left(\frac{\partial g}{\partial u_{\sigma}}\right)^{2}=4 \varepsilon^{2} \varepsilon_{k+\sigma}^{2}\left(u_{\sigma} \kappa_{\sigma}\right)^{2} \kappa_{\sigma}^{2} \quad, \quad 1 \leq \sigma \leq m
\end{gathered}
$$

and

$$
\frac{\partial g}{\partial u_{m+\rho}}=\frac{\partial^{2} g}{\partial u_{m+\rho}^{2}}=\left(\frac{\partial g}{\partial u_{m+\rho}}\right)^{2}=0 \quad, \quad 1 \leq \rho \leq k-m .
$$

Substituting these equations into the equation (3.4), we find the $\sigma^{\text {th }}$ non-degenerate principal sectional curvature to be

$$
K_{\xi}\left(e_{\sigma}, n\right)=\varepsilon_{\sigma} \kappa_{\sigma}^{2}\left(\frac{\left(u_{\sigma} \kappa_{\sigma}\right)^{2}-\varepsilon_{k+\sigma}\left(\sum_{\sigma=1}^{m} \varepsilon_{k+\sigma}\left(u_{\sigma} \kappa_{\sigma}\right)^{2}+\varepsilon_{k+m+1}\left(\eta_{m+1}\right)^{2}\right)}{\left(\sum_{\sigma=1}^{m} \varepsilon_{k+\sigma}\left(u_{\sigma} \kappa_{\sigma}\right)^{2}+\varepsilon_{k+m+1}\left(\eta_{m+1}\right)^{2}\right)^{2}}\right)
$$

where $\varepsilon=\varepsilon_{1} \cdot \varepsilon_{2} \cdot \varepsilon_{3} . \ldots \varepsilon_{k}, \varepsilon^{2}=1, \varepsilon_{\sigma}=\left\langle e_{\sigma}, e_{\sigma}\right\rangle= \pm 1, \varepsilon_{k+\sigma}=\left\langle a_{k+\sigma}, a_{k+\sigma}\right\rangle= \pm 1$, $\varepsilon_{k+m+1}=\left\langle a_{k+m+1}, a_{k+m+1}\right\rangle= \pm 1$.

After simple calculations, we reach the equation (3.5). Similarly, when we calculate the $(m+\rho)^{\text {th }}$ principal sectional curvature, we find

$$
K_{\xi}\left(e_{m+\rho}, n\right)=0 \quad, \quad 1 \leq \rho \leq k-m .
$$

Therefore, we give the following corollary.

Corollary 3.1. Let $M$ be a generalized semi-ruled surface with central ruled surface in $n$-dimensional semi-Euclidean space $E_{v}^{n+1}$. For $1 \leq \rho \leq k-m$ the $(m+\rho)^{\text {th }}$ principal sectional curvature of $M$ vanishes at the point $\xi \in M$.

Theorem 3.3. Let $M$ be a generalized semi-ruled surface with central ruled surface and $P_{\sigma}, 1 \leq \sigma \leq m$, be the $\sigma^{\text {th }}$ principal distribution parameter of $M$ in $(n+$ $1)$-dimensional semi-Euclidean space $E_{v}^{n+1}$. At the central point $\zeta \in \Omega$, the $\sigma^{\text {th }}$ 
principal sectional curvature and the $(m+\rho)^{\text {th }}$ principal sectional curvature of $M$ are

$$
\begin{gathered}
K_{\zeta}\left(e_{\sigma}, n\right)=-\varepsilon_{\sigma} \varepsilon_{k+\sigma} \varepsilon_{k+m+1}\left(\frac{\kappa_{\sigma}}{\left(\eta_{m+1}\right)}\right)^{2} \quad, \quad 1 \leq \sigma \leq m, \\
K_{\varsigma}\left(e_{m+\rho}, n\right)=0,1 \leq \rho \leq k-m,
\end{gathered}
$$

respectively.

Proof. By taking into consideration the equations (2.6) and (3.4), the $\sigma^{\text {th }}$ sectional curvature of $M$ is found as

$$
\begin{aligned}
K_{\zeta}\left(e_{\sigma}, n\right) & =\varepsilon_{\sigma} \kappa_{\sigma}^{2}\left(\frac{\left(u_{\sigma} \kappa_{\sigma}\right)^{2}-\varepsilon_{k+\sigma}\left(\sum_{\sigma=1}^{m} \varepsilon_{k+\sigma}\left(u_{\sigma} \kappa_{\sigma}\right)^{2}+\varepsilon_{k+m+1}\left(\eta_{m+1}\right)^{2}\right)}{\left(\sum_{\sigma=1}^{m} \varepsilon_{k+\sigma}\left(u_{\sigma} \kappa_{\sigma}\right)^{2}+\varepsilon_{k+m+1}\left(\eta_{m+1}\right)^{2}\right)^{2}}\right) \\
& =\varepsilon_{\sigma} \kappa_{\sigma}^{2}\left(\frac{-\varepsilon_{k+\sigma} \varepsilon_{k+m+1}\left(\eta_{m+1}\right)^{2}}{\left(\varepsilon_{k+m+1}\left(\eta_{m+1}\right)^{2}\right)^{2}}\right) \quad, \quad 1 \leq \sigma \leq m,
\end{aligned}
$$

where $\zeta \in \Omega$ is central point of $M$. After simplifying the last equation, we get

$$
K_{\zeta}\left(e_{\sigma}, n\right)=-\varepsilon_{\sigma} \varepsilon_{k+\sigma} \varepsilon_{k+m+1}\left(\frac{\kappa_{\sigma}}{\left(\eta_{m+1}\right)}\right)^{2}, \quad 1 \leq \sigma \leq m,
$$

where $\varepsilon_{k+\sigma}=\left\langle a_{k+\sigma}, a_{k+\sigma}\right\rangle= \pm 1, \varepsilon_{k+m+1}=\left\langle a_{k+m+1}, a_{k+m+1}\right\rangle= \pm 1, \varepsilon_{\sigma}=$ $\left\langle e_{\sigma}, e_{\sigma}\right\rangle= \pm 1$.

Moreover, from the Corollary 3.1, it is clear that the $(m+\rho)^{t h}$ principal sectional curvature is zero.

Let's $e$ be a unit vector in generating space $E_{k, \mu}(t)$ of ruled surface $M$ and $n$ be a non-null normal tangent vector orthogonal to $E_{k, \mu}(t)$ of $M$. Now, we can investigate the curvature of tangential section $(e, n)$.

Since the unit vector $e(t)$ is in $E_{k, \mu}(t)$, we write

So,

$$
e(t) \in S p\left\{e_{1}(t), \ldots, e_{m}(t), e_{m+1}(t), \ldots, e_{k}(t)\right\}
$$

$$
e(t)=\sum_{x=1}^{s} \lambda_{x} e_{x}(t)+\sum_{y=s+1}^{m} \lambda_{y} e_{y}(t)+\sum_{z=m+1}^{m+1+\mu-s} \lambda_{z} e_{z}(t)+\sum_{w=m+2+\mu-s}^{k} \lambda_{w} e_{w}(t)
$$

where $\|e(t)\|=1$.

On account of $E_{k, \mu}(t)=S p\left\{e_{1}(t), \ldots, e_{k}(t)\right\}$, due to $F_{m, s}(t)=S p\left\{e_{1}(t), \ldots\right.$, $\left.e_{m}(t)\right\}$ and $Z_{k-m, \mu-s}(t)=S p\left\{e_{m+1}(t), \ldots, e_{k}(t)\right\}$, there are spacelike and timelike vectors in the subspace $F_{m, s}(t)$ and $Z_{k-m, \mu-s}(t)$. We assume that the numbers of timelike vectors be $s$ and $\mu-s$ in $F_{m, s}(t)$ and $Z_{k-m, \mu-s}(t)$, respectively. In this case, both $F_{m, s}(t)$ and $Z_{k-m, \mu-s}(t)$ are semi-subspaces. Thus, the central ruled surface of generalized ruled surface is semi-subspace. Furthermore, the unit vector $e(t)$ is non-null. 
Therefore there exist the following two cases. Now we consider these situations, separately.

1. Case: We can write

$$
e=\sum_{i=1}^{s} \sinh \theta_{i} e_{i}+\sum_{j=s+1}^{m} \cosh \theta_{j} e_{j}+\sum_{l=m+1}^{m+\mu-s} \sinh \theta_{l} e_{l}+\sum_{w=m+1+\mu-s}^{k} \cosh \theta_{w} e_{w} .
$$

It is clear here that

$$
-\sum_{i=1}^{s} \sinh ^{2} \theta_{i}+\sum_{j=s+1}^{m} \cosh ^{2} \theta_{j}-\sum_{l=m+1}^{m+\mu-s} \sinh ^{2} \theta_{l}+\sum_{w=m+1+\mu-s}^{k} \cosh ^{2} \theta_{w}=1
$$

where the angles $\theta_{1}, \theta_{2}, \ldots, \theta_{s}, \ldots, \theta_{k}$ are hyperbolic angles between spacelike unit vector $e$ and the base vectors $e_{1}, e_{2}, \ldots, e_{s}, \ldots, e_{k}$, respectively.

2. Case: The unit vector $e(t)$ is timelike

The unit vector $e$ can be written

$$
e=\sum_{i=1}^{s} \cosh \theta_{i} e_{i}+\sum_{j=s+1}^{m} \sinh \theta_{j} e_{j}+\sum_{l=m+1}^{m+\mu-s} \cosh \theta_{l} e_{l}+\sum_{w=m+1+\mu-s}^{k} \sinh \theta_{w} e_{w} .
$$

From the above equation, it is seen that

$$
-\sum_{i=1}^{s} \sinh ^{2} \theta_{i}+\sum_{j=s+1}^{m} \cosh ^{2} \theta_{j}-\sum_{l=m+1}^{m+\mu-s} \sinh ^{2} \theta_{l}+\sum_{w=m+1+\mu-s}^{k} \cosh ^{2} \theta_{w}=-1
$$

where the angles $\theta_{1}, \theta_{2}, \ldots, \theta_{s}, \ldots, \theta_{k}$ are hyperbolic angles between timelike unit vector $e$ and the base vectors $e_{1}, e_{2}, \ldots, e_{s}, \ldots, e_{k}$, respectively.

Thus, we can give the following theorems for the curvatures of tangential section $(e, n)$ for these two cases, respectively.

Theorem 3.4. Let $M$ be a generalized semi-ruled surface with central ruled surface in $(n+1)$-dimensional semi-Euclidean space $E_{v}{ }^{n+1}$ and e be a spacelike unit vector in $E_{k, \mu}(t)$, taking $n$ to be a non-null normal tangential vector orthogonal to $E_{k, \mu}(t)$ of $M$. There exists the following relation between the sectional curvature of non-degenerate section $(e, n)$ and principal sectional curvatures at the point $\zeta \in \Omega \subset M$

$$
K_{\zeta}(e, n)=-\sum_{i=1}^{s} \sinh ^{2} \theta_{i} K_{\zeta}\left(e_{i}, n\right)+\sum_{j=s+1}^{m} \cosh ^{2} \theta_{j} K_{\zeta}\left(e_{j}, n\right)
$$

where $e_{s}, 1 \leq s \leq m$, are a timelike vectors in subspace $F_{m, s}(t)$ and the hyperbolic angles between spacelike unit vector $e$ and the base vectors $e_{1}, e_{2}, \ldots, e_{s}$, $e_{s+1}, \ldots, e_{m}, e_{m+1}, \ldots, e_{k}$ are $\theta_{1}, \theta_{2}, \ldots, \theta_{s}, \theta_{s+1}, \ldots, \theta_{m}, \theta_{m+1}, \ldots, \theta_{k}$, respectively.

Proof. Let the coordinates of the spacelike unit vector $e$ within the generating space $E_{k}, \mu(t)$ be $\left(\beta_{0}, \beta_{1}, \ldots, \beta_{k}\right)$ and the coordinates of non-null normal tangent vector $n$ be $\left(\gamma_{0}, \gamma_{1}, \ldots, \gamma_{k}\right)$. From the equations (3.1) and (3.2), at the central point $\zeta \in \Omega$, we obtain 


$$
K_{\zeta}(e, n)=\frac{\sum_{i=1}^{s} \sinh ^{2} \theta_{i} R_{i 0 i 0}+\sum_{j=s+1}^{m} \cosh ^{2} \theta_{j} R_{i 0 i 0}}{\langle e, e\rangle\langle n, n\rangle-\langle e, n\rangle^{2}} .
$$

If we take into consideration the last equation and the equation (2.9), we find

$$
K_{\zeta}(e, n)=\sum_{i=1}^{s} \sinh ^{2} \theta_{i}\left(-\frac{1}{2 g} \frac{\partial^{2} g}{\partial u_{i}{ }^{2}}+\frac{1}{4 g^{2}}\left(\frac{\partial g}{\partial u_{i}}\right)^{2}\right)+\sum_{j=s+1}^{m} \cosh ^{2} \theta_{j}\left(-\frac{1}{2 g} \frac{\partial^{2} g}{\partial u_{i}{ }^{2}}+\frac{1}{4 g^{2}}\left(\frac{\partial g}{\partial u_{i}}\right)^{2}\right) .
$$

By considering the equation (3.4) at the central point $\zeta \in \Omega$, a relation between the sectional curvature of section $(e, n)$ and principal sectional curvatures of $M$ given in (3.11) is obtained. Thus, the proof is completed.

This relation is called I. type semi-Euclidean Beltrami-Euler formula for the section of generalized semi-ruled surface with central ruled surface at the central point $\zeta \in \Omega$.

Theorem 3.5. Let $M$ be a generalized semi-ruled surface with central ruled surface

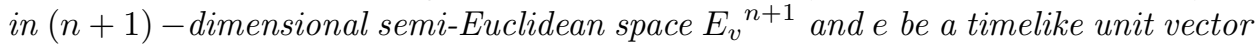
in $E_{k, \mu}(t)$, taking $n$ to be a non-null normal tangential vector orthogonal to $E_{k, \mu}(t)$ of $M$. There is the following relation between sectional curvature of non-degenerate section $(e, n)$ and principal sectional curvatures at the point $\zeta \in \Omega \subset M$

$$
K_{\zeta}(e, n)=\sum_{i=1}^{s} \cosh ^{2} \theta_{i} K_{\zeta}\left(e_{i}, n\right)-\sum_{j=s+1}^{m} \sinh ^{2} \theta_{j} K_{\zeta}\left(e_{j}, n\right)
$$

where $e_{s}, 1 \leq s \leq m$, are a timelike vectors in subspace $F_{m, s}(t)$ and the angles $\theta_{1}, \theta_{2}, \ldots, \theta_{s}, \theta_{s+1}, \ldots, \theta_{m}, \theta_{m+1}, \ldots, \theta_{k}$ are hyperbolic angles between the timelike unit vector $e$ and the base vectors $e_{1}, e_{2}, \ldots, e_{s}, e_{s+1}, e_{m}, e_{m+1}, \ldots, e_{k}$, respectively.

Proof. Let the coordinates of the timelike unit vector $e$ in $E_{k, \mu}(t)$ be $\left(\beta_{0}, \beta_{1}, \ldots, \beta_{k}\right)$ and the coordinates of non-null normal tangent vector $n$ be $\left(\gamma_{0}, \gamma_{1}, \ldots, \gamma_{k}\right)$. From the equation (3.1), at the central point $\zeta \in \Omega$, we find

$$
K_{\zeta}(e, n)=\frac{\sum_{i=1}^{s} \cosh ^{2} \theta_{i} R_{i 0 i 0}+\sum_{j=s+1}^{m} \sinh ^{2} \theta_{j} R_{j 0 j 0}}{\langle e, e\rangle\langle n, n\rangle-\langle e, n\rangle^{2}} .
$$

Taking into consideration the last equation and the equation (2.9), we obtain

$$
K_{\zeta}(e, n)=-\sum_{i=1}^{s} \cosh ^{2} \theta_{i}\left(-\frac{1}{2 g} \frac{\partial^{2} g}{\partial u_{i}{ }^{2}}+\frac{1}{4 g^{2}}\left(\frac{\partial g}{\partial u_{i}}\right)^{2}\right)-\sum_{j=s+1}^{m} \sinh ^{2} \theta_{j}\left(-\frac{1}{2 g} \frac{\partial^{2} g}{\partial u_{i}{ }^{2}}+\frac{1}{4 g^{2}}\left(\frac{\partial g}{\partial u_{i}}\right)^{2}\right) .
$$

By considering the equation (3.4) at the central point $\zeta \in \Omega$, a relation between the sectional curvature of the section $(e, n)$ and principal sectional curvatures of $M$ given in (3.12) is obtained. Thus, the proof is completed.

This relation is called II. type semi-Euclidean Beltrami-Euler formula for the section of generalized semi-ruled surface with central ruled surface at the central point $\zeta \in \Omega$. 


\section{REFERENCES}

[1] Akyigit, M., Ersoy, S. and Tosun, M., Beltrami-Meusnier formulas of generalized semi ruled surfaces in semi Euclidean space, Kuwait J. Sci. 41(2) (2014), 65-83.

[2] Beem, J. K., Ehrlich, P. E. and Easley, K. L., Global Lorentzian Geometry, Marcel Dekker, New York, 1981.

[3] Coken, A. C. Ciftci, U. and Ekici, C., On parallel timelike ruled surfaces with timelike rulings, Kuwait J. Sci. 1A (2008), 21-32.

[4] Ekici, C. and Görgülü, A., On the curvatures of $(k+1)$-dimensional semi ruled surfaces in $E_{v}^{n+1}$, Math. Comput. Appl. 5(3) (2000), 139-148.

[5] Ersoy, S. and Tosun, M., Lorentzian Beltrami-Euler formula and generalized Lorentzian Lamarle formula in $I R_{1}^{n}$, Matematiche (Catania) 64(1) (2009), 25-45.

[6] Ersoy, S. and Tosun, M., Sectional curvature of timelike ruled surface Part I: Lorentzian Beltrami-Euler formula, Iran. J. Sci. Technol. Trans. A Sci. 34(A3) (2010), 197-214.

[7] Ersoy, S. and Tosun, M., Lorentzian Beltrami-Meusnier Formula, Gen. Math. Notes. 18 (2013), 64-87.

[8] Frank, H. and Giering, O., Verallgemeinerte regelflächen, Math. Z. 150 (1976), 261-271.

[9] Frank, H. and Giering, O., Zur Schnittkrümmung verallgemeinerter Regelflächen, Arch. Math. Fasc.1, 32 (1979), 86-90.

[10] O’Neill, B., Semi Riemannian Geometry, Academic Press, New York, 1983.

[11] Thas, C., Properties of ruled surfaces in the Euclidean space $E^{n}$, Bull. Inst. Math. Acad. Sinica. 6(1) (1978), 133-142.

[12] Tosun, M. and Kuruog̃lu, N., On $(k+1)$-dimensional time-like ruled surface in the Minkowski space $\mathbb{R}_{1}^{n}$, J. Inst. Math. Comput. Sci. Math. Ser. 11(1) (1998), 1-9.

[13] Yoon, D. W. and Park, J. H., On curvatures of ruled surfaces in Minkowski 3-spaces, Note Mat., 2(2) (2008) 43-56.

Department of Mathematics, Sakarya University, Sakarya, Turkey

E-mail address: makyigit@sakarya.edu.tr

E-mail address: sersoy@sakarya.edu.tr

E-mail address: tosun@sakarya.edu.tr 